\title{
A Countable Economy: An Example
}

\author{
Attila Tasnádi \\ Department of Mathematics, Corvinus University of Budapest, H-1093 \\ Budapest, Fővám tér 8, Hungary \\ October 24, 2004. \\ Electronic version of an article published as \\ International Game Theory Review, Volume 8, Issue 4, 2006, 555-560 \\ doi: 10.1142/S0219198906001107 \\ (C) Copyright World Scientific Publishing Company \\ URL: IGTR
}

\begin{abstract}
Weiss (1981) established core equivalence and the existence of competitive equilibria in finitely additive exchange economies. To underline the relevance of finitely additive economies we present in this note an example with a close connection to finite exchange economies.
\end{abstract}

JEL Classification Number: D51.

Keywords: Core equivalence; Countable economies; Asymptotic density.

\section{Introduction}

In his path breaking work Aumann (1964) gave an exact mathematical model for pure competition by taking a continuum of traders. His result was generalized in many ways. For instance, Hildenbrand (1974) extended Aumann's (1964) result to atomless $\sigma$-additive exchange economies. Weiss (1981) went even further in considering finitely additive exchange economies. One main point is that the finitely additive framework allows the set of traders to be only countable.

Weiss (1981) and Armsrtrong and Richter (1984) provided several examples for just finitely additive economies. In this note we consider an example with a close connection to finite exchange economies. Taking a finite exchange economy with traders $\{1, \ldots, n\}$, the average endowments and the average allocations are determined by the arithmetic means of the individual endowments $\left(\omega_{i}\right)_{i=1}^{n}$ and the individual allocations $\left(x_{i}\right)_{i=1}^{n}$, respectively. Thus, if we

\footnotetext{
${ }^{*}$ Telephone: (+36 1) 4828782, E-mail: attila.tasnadi@uni-corvinus.hu
} 
identify the set of traders with the set of positive integers, it seems to be natural to express the average endowments by $\lim _{n \rightarrow \infty} \frac{1}{n} \sum_{i=1}^{n} \omega_{i}$ and the average allocations by $\lim _{n \rightarrow \infty} \frac{1}{n} \sum_{i=1}^{n} x_{i}$. However, these limits do not exist for arbitrary sequences. Brown and Robinson (1975) overcame this type of difficulty by considering a non-standard exchange economy, while we remain in the framework of a standard exchange economy and define the core following Weiss (1981) 11 By considering this example, we want to emphasize the importance of the finitely additive framework. For this example core equivalence and the non-emptiness of the core follows from Weiss (1981).

\section{Preliminaries}

We denote the set of positive integers by $\mathbf{N}$, and the set of real numbers by $\mathbf{R}$. For $M \subset \mathbf{N}$, write $\mathbf{1}_{M}$ for the characteristic function of $M$; that is, the function which is 1 on $M$ and 0 elsewhere. Fix an arbitrary set $M \subset$ $\mathbf{N}$. We say that the sequence $f: \mathbf{N} \rightarrow \mathbf{R}$ is $M$-summable if the sequence $\frac{1}{n} \sum_{i=1}^{n} \mathbf{1}_{M}(i) f(i)$ is convergent. For an $M$-summable $f$ let us introduce the notation

$$
s(f, M):=\lim _{n \rightarrow \infty} \frac{1}{n} \sum_{i=1}^{n} \mathbf{1}_{M}(i) f(i) .
$$

We call the value $s(f, M)$ the $M$-sum of sequence $f$. $\mathbf{N}$-summable sequences are usually called Cesàro summable sequences. Hence, $f$ is $M$-summable if $1_{M} f$ is Cesàro summable. Since not every bounded sequence is $M$-summable, we will extend the notion of $M$-sum. For the extension we will employ the Banach Limit. In the following let $T$ be an arbitrary but fixed Banach Limit on $\ell_{\infty}$. Let

$$
s_{n}(f, M):=\frac{1}{n} \sum_{i=1}^{n} \mathbf{1}_{M}(i) f(i), \quad M \subset \mathbf{N} .
$$

Definition 1. For any set $M \subset \mathbf{N}$ and any sequence $f: \mathbf{N} \rightarrow \mathbf{R}$ for which $\left(s_{n}(f, M)\right)_{n \in \mathbf{N}} \in \ell_{\infty}$ holds true we define the $M$-sum of sequence $f$ by

$$
s(f, M):=T\left(\left(s_{1}(f, M), s_{2}(f, M), \ldots\right)\right) .
$$

Note that if $f \in \ell_{\infty}$, then $s(f, M)$ is defined for any set $M \subset \mathbf{N}$. It is also easy to see that $s(f, \cdot)$ is a finitely additive measure for any fixed $f \in \ell_{\infty}$. We are looking for the finitely additive measure $\nu$ for which $s(f, M)$ obtains

\footnotetext{
${ }^{1}$ In another paper, Tasnádi (2002), we worked with a different definition of the core in which case the core equivalence theorems found in the literature could not be applied.
} 
as $\int_{M} f d \nu$. As it will turn out, $\nu$ will be also useful in defining the size of a coalition.

Our aim is to allow any $M$ subset of $\mathbf{N}$ as a possible coalition. Let $\mathcal{D}$ consist of those sets $M$ subsets of $\mathbf{N}$ for which $\lim _{n \rightarrow \infty} \frac{1}{n} \sum_{i=1}^{n} \mathbf{1}_{M}(i)$ exists. Let us define the asymptotic density, henceforth briefly density, of a set of positive integers $M \in \mathcal{D}$ by

$$
\nu(M):=\lim _{n \rightarrow \infty} \frac{1}{n} \sum_{i=1}^{n} \mathbf{1}_{M}(i) .
$$

The density cannot be defined on every subset of $\mathbf{N}$ by expression (1). Examples of sets for which the limit in expression (1) does not exist can be found for instance in Buck (1946). The density, defined so far on $\mathcal{D}$, can be extended to the class $\mathcal{P}(\mathbf{N})$, where $\mathcal{P}(\mathbf{N})$ denotes the power set of $\mathbf{N}$ (see for instance Buck, 1946). We define the sequence of measures $\left(\nu_{n}\right)_{n \in \mathbf{N}}$ on $\mathcal{P}(\mathbf{N})$ in the following way:

$$
\nu_{n}(M):=\frac{1}{n} \sum_{i=1}^{n} \mathbf{1}_{M}(i), \quad M \subset \mathbf{N} .
$$

Definition 2. We define the density of a subset $M$ of $\mathbf{N}$ by

$$
\nu(M):=T\left(\left(\nu_{1}(M), \nu_{2}(M), \ldots\right)\right),
$$

where $T$ denotes the same Banach Limit employed in the extension of $s$.

The density $\nu$ is a finitely additive measure on $(\mathbf{N}, \mathcal{P}(\mathbf{N}))$. We will show in Proposition 1 that the integral of a sequence with respect to the density plays a special role because it equals in case of a bounded sequence a very natural 'infinite average' of the sequence.

Weiss (1981) described the theory of measurability and integration for finite and nonnegative measures when the underlying algebra consists of all subsets of the base set. A subset $A$ of $\mathbf{N}$ is called a null set if $\nu(A)=0$. We say that a property holds for almost all $\mathbf{N}$ if this property is only violated on a null subset of $\mathbf{N}$. A function $h: \mathbf{N} \rightarrow \mathbf{R}$ is said to be a null function if $\nu(\{n \in \mathbf{N}:|h(n)|>\alpha\})=0$ for each $\alpha>0$. The norm of a function $f: \mathbf{N} \rightarrow \mathbf{R}$ is defined by

$$
\|f\|_{*}:=\inf _{\alpha>0}\{\alpha+\nu(\{n \in \mathbf{N}:|f(n)|>\alpha\})\} .
$$

A function $h: \mathbf{N} \rightarrow \mathbf{R}$ is a null function if and only if $\|h\|_{*}=0$. A sequence $\left(f_{n}\right)$ of functions on $\mathbf{N}$ to $\mathbf{R}$ converges in density to the function $f$ on $\mathbf{N}$ to $\mathbf{R}$ 
if and only if $\lim _{n \rightarrow \infty}\left\|f_{n}-f\right\|_{*}=0$. A function $f: \mathbf{N} \rightarrow \mathbf{R}$ is called simple if there exists a function $g: \mathbf{N} \rightarrow \mathbf{R}$ which has only a finite set of values and a null function $h$ so that $f=g+h$. A function $f: \mathbf{N} \rightarrow \mathbf{R}$ is measurable if there exists a sequence $\left(f_{n}\right)$ of simple functions converging in density to $f$. By Weiss (1981) Lemma 5 every function $f: \mathbf{N} \rightarrow \mathbf{R}$ is measurable.

Now we can prove the relationship between $M$-sums and the density.

Proposition 1. If $f \in \ell_{\infty}$ and $M \subset \mathbf{N}$, then $s(f, M)=\int_{M} f d \nu$.

Proof. First, let $f$ be a bounded simple sequence. Hence, $f=g+h$, where sequence $g$ takes only values $\left\{x_{1}, \ldots, x_{l}\right\}$ and $h$ is a bounded null sequence. Let $E_{i}:=g^{-1}\left(\left\{x_{i}\right\}\right)$, where $i \in\{1, \ldots, l\}$. The following equalities show us the identity of the integral and the $\mathbf{N}$-sum for simple sequences

$$
\begin{aligned}
\int f d \nu & =\int g d \nu=\sum_{i=1}^{l} x_{i} \nu\left(E_{i}\right)=\sum_{i=1}^{l} x_{i} T\left(\left(\nu_{1}\left(E_{i}\right), \nu_{2}\left(E_{i}\right), \ldots\right)\right)= \\
& =\sum_{i=1}^{l} x_{i} T\left(\left(s_{1}\left(\mathbf{1}_{E_{i}}, \mathbf{N}\right), s_{2}\left(\mathbf{1}_{E_{i}}, \mathbf{N}\right), \ldots\right)\right)= \\
& =T\left(\left(\sum_{i=1}^{l} x_{i} s_{1}\left(\mathbf{1}_{E_{i}}, \mathbf{N}\right), \sum_{i=1}^{l} x_{i} s_{2}\left(\mathbf{1}_{E_{i}}, \mathbf{N}\right), \ldots\right)\right)= \\
& =T\left(\left(s_{1}(g, \mathbf{N}), s_{2}(g, \mathbf{N}), \ldots\right)\right)=s(g, \mathbf{N})=s(f, \mathbf{N}) .
\end{aligned}
$$

Now take any bounded sequence $f$. Then there exists a uniformly bounded sequence $\left(f_{n}\right)$ of simple functions converging in density to $f$. Let $K$ be a uniform bound for sequences $\left|f_{n}\right|$ and $|f|$. We obtain by the dominated convergence theorem that $f$ is integrable and that $\lim _{n \rightarrow \infty} \int\left|f-f_{n}\right| d \nu=0$. Therefore, it follows that

$$
\int f d \nu=\lim _{n \rightarrow \infty} \int f_{n} d \nu=\lim _{n \rightarrow \infty} s\left(f_{n}, \mathbf{N}\right) .
$$

To any positive value $\varepsilon$ there exists an index $n_{0}$ such that

$$
\nu\left(\left\{i \in \mathbf{N}:\left|f(i)-f_{n}(i)\right|>\varepsilon\right\}\right)<\varepsilon
$$

for all $n \geq n_{0}$. Let $A_{\varepsilon, n}:=\left\{i \in \mathbf{N}:\left|f(i)-f_{n}(i)\right|>\varepsilon\right\}$. Then we can write

$$
\begin{aligned}
s\left(\left|f-f_{n}\right|, \mathbf{N}\right) & =s\left(\left|f-f_{n}\right|, A_{\varepsilon, n}\right)+s\left(\left|f-f_{n}\right|, A_{\varepsilon, n}^{c}\right) \leq \\
& \leq 2 K \nu\left(A_{\varepsilon, n}\right)+\varepsilon \nu\left(A_{\varepsilon, n}^{c}\right)<(2 K+1) \varepsilon
\end{aligned}
$$

for all $n \geq n_{0}$. By (3) it can be easily checked that $s(f, \mathbf{N})=$ $\lim _{n \rightarrow \infty} s\left(f_{n}, \mathbf{N}\right)$. Thus, regarding (2), we have established that $\int f d \nu=$ $s(f, \mathbf{N})$. Clearly, this identity also holds for sequences of type $1_{M} f$. 
The following example demonstrates that in Proposition 1 the assumption that the sequence must be bounded cannot be dropped.

Example 1. Let us consider the sequence $f(n):=n$ if $\log _{2} n \in \mathbf{N} \cup\{0\}$, and $f(n):=0$ elsewhere. It can be checked that $s(f, \mathbf{N}) \in[1,2]$, while $\int f d \nu=0$.

\section{The countable economy}

The commodity space is $\mathbf{R}^{d}$, where $d$ stands for the number of different commodities being traded in the market. The symbol $\theta$ denotes the origin in $\mathbf{R}^{d}$. Superscripts will be used to denote coordinates. For $x, y \in \mathbf{R}^{d}$ we write $x>y$ if $x^{i}>y^{i}$ for all $i=1, \ldots, d$, and $x \geq y$ if $x^{i} \geq y^{i}$ for all $i=1, \ldots, d$. The consumption set is $\mathbf{R}_{+}^{d}:=\left\{x \in \mathbf{R}^{d} \mid x \geq \theta\right\}$. The set of price vectors is $P:=\mathbf{R}_{+}^{d} \backslash\{\theta\}$. A preference relation $\succ \subset \mathbf{R}_{+}^{d} \times \mathbf{R}_{+}^{d}$ is a transitive and irreflexive binary relation on $\mathbf{R}_{+}^{d}$. The set of traders is $\mathbf{N}$. We assume that the traders' preferences $\left(\succ_{n}\right)_{n \in \mathbf{N}}$ satisfy monotonicity, i.e., for all $n \in \mathbf{N}$ and all $x, y \in \mathbf{R}_{+}^{d}$ from $x \geq y$ and $x \neq y$ it follows that $x \succ_{n} y$. Each trader $n \in \mathbf{N}$ possesses an initial endowment $\omega(n) \in \mathbf{R}_{+}^{d}$. An exchange economy is given by the traders' preferences and initial endowments.

We follow Weiss (1981) in defining the core of our exchange economy. A nonempty set $I \subset \mathbf{N}$ is called a coalition. A null coalition is a coalition of a null set of traders. An assignment is an integrable vector-valued sequence $f: \mathbf{N} \rightarrow \mathbf{R}_{+}^{d}$ and an allocation $f$ is an assignment such that $\int f d \nu=\int \omega d \nu$. We define for any nonnull coalition $I$ the coalitional preference $\succ_{I}$, which is defined for any pair of assignments, in the following way:

$$
f \succ_{I} g \quad \Leftrightarrow \quad f(i) \oplus h_{1}(i) \succ_{i} g(i) \oplus h_{2}(i),
$$

for all null functions $h_{1}, h_{2}$, for almost all $i \in I$, where

$$
(x \oplus y)^{k}:=\max \left\{x^{k}+y^{k}, 0\right\}, \quad k=1, \ldots, d .
$$

The nonnull coalition $I \subset \mathbf{N}$ improves upon an assignment $g$ with an assignment $f$ if $f \succ_{I} g$ and $\int_{I} f d \nu=\int_{I} \omega d \nu$.

Definition 3. We define the core as the set of those allocations which cannot be improved upon via any nonnull coalition.

We define the competitive equilibrium also in line with Weiss (1981). We say that an assignment $f$ is in the budget set of $I$ if there exists a real-valued null sequence $h$ such that for almost all $i \in I$ we have $p f(i)-h(i) \leq p \omega(i)$. An assignment $f$ is a maximal assignment in the budget set of $I$ if $f$ is in the budget set of $I$ and if for every assignment $g$ it follows from $g \succ_{I} f$ that $g$ is not contained in the budget set of $J$ for any nonnull subcoalition $J \subset I$. 
Definition 4. We say that an allocation $f$ is a competitive equilibrium allocation if there exists a price vector $p \in P$ such that $f$ is a maximal assignment in the budget set of $I$ for any nonnull coalition $I$.

To apply Weiss's (1981) core equivalence theorem and existence theorem we only have to impose an additional assumption and check that $\nu$ is nonatomic.

Assumption 1. Suppose that $z \in \mathbf{R}^{d}, z>\theta, h: \mathbf{N} \rightarrow \mathbf{R}^{d}$ is a null function and $I \subset \mathbf{N}$ is a nonnull coalition. Then $g(i) \succ_{i} f(i)$ for almost all $i \in I$ implies

$$
\nu\left(i \in I \mid g(i)+z \succ_{i} f(i)+h(i)\right)=\nu(i \in I \mid f(i)+h(i) \geq \theta) .
$$

Maharam (1976) established that $\nu$ is full valued, i.e., to any set $B \subset \mathbf{N}$ with positive density $\beta$ and to any real value $\alpha \in[0, \beta]$, there exists a subset $A$ of $B$ with density $\alpha$. This clearly implies that $\nu$ is nonatomic, i.e., for any subset $B$ of $\mathbf{N}$ and for any $\eta>0$ there exists a subset $A$ of $B$ such that $|\nu(B) / 2-\nu(A)|<\eta$. Hence, by applying Weiss (1981) Theorems 2 and 3 we obtain the following theorem.

Theorem 1. If Assumption 1 is satisfied and $\int \omega d \nu>0$, then the core coincides with the nonempty set of competitive allocations.

\section{Acknowledgments}

I would like to thank Péter Tallos for his helpful suggestions. This research was done during the author's Bolyai János Research Fellowship provided by the Hungarian Academy of Sciences (MTA).

\section{References}

[1] Armstrong, Thomas E. and Marcel K. Richter, (1984). "The Core-Walras Equivalence," Journal of Economic Theory Vol. 33, No. 1, 116-151.

[2] Aumann, Robert J. (1964). "Market with a Continuum of Traders," Econometrica Vol. 32, No. 1-2, 39-50.

[3] Brown, Donald J. and Abraham Robinson (1975). "Nonstandard Exchange Economies," Econometrica Vol .43, No. 1, 41-55.

[4] Buck, R. Creighton (1946). "The Measure Theoretic Approach to Density," American Journal of Mathematics Vol. 68, 560-580. 
[5] Hildenbrand, Werner (1974). "Core and Equilibria of a Large Economy," Princeton University Press.

[6] Maharam, Dorothy (1976). "Finitely additive measures on the integers," Sankhyā, Series A Vol. 38, No. 1, 44-59.

[7] Tasnádi, Attila (2002). "An elementary core equivalence theorem in a countable economy," Decisions in Economics and Finance Vol. 25, No. 2, 127-143.

[8] Weiss, Ernst-August Jr. (1981). "Finitely Additive Exchange Economies," Journal of Mathematical Economics Vol. 8, No. 2, 221-240. 